\title{
A structured patient empowerment programme for primary immunodeficiency significantly improves general and health-related quality of life
}

\author{
MARIA FASSHAUER ${ }^{I *}$, GESINE SCHUERMANN ${ }^{2 *}$, NORBERT GEBERT ${ }^{3}$, \\ HORST VON BERNUTH ${ }^{4}$, SIGUNE GOLDACKER ${ }^{5}$, RENATE KRUEGER ${ }^{4}$, PETRA MANZEY, \\ GUNDULA NOTHEIS ${ }^{7}$, HENRIKE RITTERBUSCH ${ }^{5}$, UWE SCHAUER ${ }^{8}$, ILKA SCHULZE, \\ VOLKER UMLAUF ${ }^{10}$, STEFFI WIDMANN ${ }^{6}$, ULRICH BAUMANN ${ }^{2}$ \\ *Maria Fasshauer and Gesine Schuermann equally contributed to this work.
}

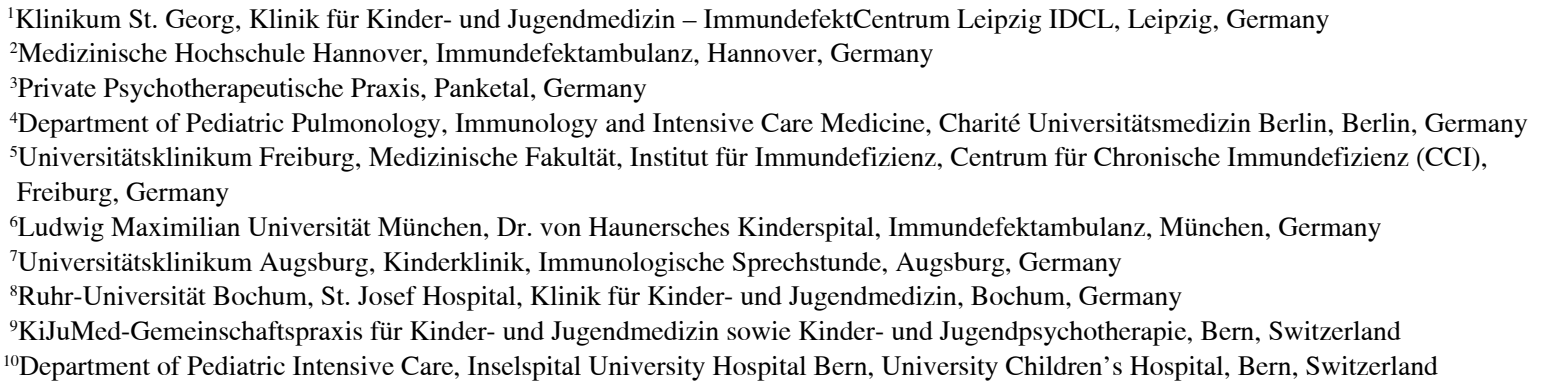

\begin{abstract}
Introduction: Primary immunodeficiencies (PIDs) are a heterogeneous group of rare diseases characterized by increased susceptibility to infections and a reduced quality of life $(Q o L)$. The influence of a patient empowerment programme for PID (PID-PEP) on general and health-related QoL was assessed in the present study.

Material and methods: PID-PEP is provided by a multidisciplinary team for patients with PID and immunoglobulin $G(\mathrm{Ig} G)$ replacement therapy during a weekend course to improve patient self-management regarding chronic disease and long-term therapy. Twenty-six adult patients with PID undergoing PID-PEP were recruited. Short Form-36 (SF-36) and the Life Quality Index (LQI) were assessed as generic and disease-specific QoL instruments before as well as 6 months after the programme.

Results: Median visual analogue scale (VAS) values of present health status significantly increased from 68 at baseline to 76 after PID-PEP $(p=0.002)$. Furthermore, the SF-36 mental component summary (MCS) significantly improved from 36 to 43 following the programme $(p=0.042)$. Of the eight $S F-36$ dimensions, vitality $(V T)$ significantly improved $(p=0.025)$. Median LQI index significantly increased from 77 at baseline to 86 after PID-PEP $(p=0.008)$. Furthermore, the LQI domains treatment interference (I) and therapy-related problems (II) significantly improved.

Conclusions: Our PID-PEP significantly improved general and health-related QoL. It needs to be evaluated in future studies whether the beneficial effects of PID-PEP are sustained over longer periods of time and whether repeated PID-PEP sessions further improve QoL outcome.
\end{abstract}

Key words: education programme, IgG replacement therapy, patient empowerment programme, primary immunodeficiency, treatment satisfaction, quality of life.

(Cent Eur J Immunol 2021; 46 (2): 244-249)

\section{Introduction}

Primary immunodeficiencies (PIDs) are a heterogeneous group of rare diseases currently comprising $>400$ distinct disorders with 430 different gene defects listed [1].
PIDs affect all parts of the immune system, are mainly characterized by increased susceptibility to infections and can be associated with autoimmune diseases [2]. The largest subgroup of PIDs is characterized by impaired B-cell

Correspondence: Dr. Maria Fasshauer, Klinikum St. Georg, Klinik für Kinder- und Jugendmedizin - ImmundefektCentrum Leipzig IDCL, Delitzscher Str. 141, 04129 Leipzig, Germany, e-mail: fasshauerm@ hotmail.com Submitted: 19.06.2020; Accepted: 14.02.2021 
development and/or maturation resulting in antibody deficiency. This PID subgroup is treated with intravenous or subcutaneous immunoglobulin $\mathrm{G}$ (IgG) replacement therapy to prevent severe infections and organ damage [2]. Early-diagnosed, IgG-treated PID patients can expect an almost normal life span; however, morbidity is substantially increased as compared to healthy controls [3]. There is a broad consensus that quality of life (QoL) is significantly decreased in PID patients even when compared to patients with other chronic diseases [4].

Various instruments are commonly used in clinical settings to assess QoL. On one hand, generic, non-disease-specific QoL assessment tools measure health status applicable to all populations. Short Form-36 (SF-36) is a frequently used generic questionnaire which contains 36 items. It results in eight health dimensions providing two summary measures, i.e. the physical component summary (PCS) and the mental component summary (MCS) $[5,6]$. On the other hand, disease-specific tools are used to assess specific aspects of chronic diseases and their treatment options. For PID, the Life Quality Index (LQI) is a well-established questionnaire evaluating QoL and treatment satisfaction in IgG replacement therapy. It contains 15 items and results in four domains providing one summary measure, i.e. the LQI index [7, 8].

Stress, recurrent infectious episodes, social determinants, and chronic health issues negatively affect QoL in PID patients [9]. All of these factors can potentially be improved by patient empowerment programmes (PEP) through which people gain greater control over decisions and actions affecting their health [10]. So far, no PEP has been established for PID patients. Therefore, a working group to develop and evaluate a primary immunodeficiency-patient empowerment programme (PID-PEP) was established in 2007 in Germany by members of the Working Group Pediatric Immunology (Arbeitsgemeinschaft Pädiatrische Immunologie - API). Changes in QoL and treatment satisfaction before and 6 months after participation in PID-PEP were evaluated in 26 adult PID patients. We hypothesized that PID-PEP would significantly improve both general (SF-36) and health-related (LQI) QoL.

\section{Material and methods}

\section{Patients}

Quality of life and treatment satisfaction were assessed before and 6 months after participating in PID-PEP in 26 adult PID patients receiving life-long IgG replacement therapy. The study period was from 2009 to 2012 . Baseline characteristics of the study population are summarized in Table 1.

\section{PID-PEP}

PID-PEP is provided by a multidisciplinary team consisting of physicians specialized in PID, psychologists, and nurses for patients with PID and IgG replacement therapy. The programme is offered near German immunodeficiency centres during a weekend group training course. Between 4 and 7 patients, as well as their relatives, participate in each course. PID-PEP consists of three main modules, i.e. I) Team building and goal definition, V) Empowerment and coping strategies, and VI) Everyday transfer of strategies learned. Furthermore, the three disease-specific modules are: II) Education on chronic disease (PID), III) Competences and motivation for long-term therapy, and IV) Management of acute and emergency situations. A more detailed description of PID-PEP is given in the Appendix. The German curriculum of PID-PEP is available online [11]. The study was approved by the ethics com-

Table 1. Baseline characteristics of the study population. Values for median (range) or total number (percentage) are shown

\begin{tabular}{lc}
\hline Variable & All subjects $(\boldsymbol{n}=\mathbf{2 6})$ \\
\hline Gender (M/F) & $8 / 18(30.8 / 69.2)$ \\
\hline Age (years) & $46.5(21-68)$ \\
\hline Duration of IgG treatment (years) & $3.5(0-38)$ \\
\hline Caucasian & $26(100)$ \\
\hline Highest qualification & $7(26.9)$ \\
\hline College or University degree & $5(19.2)$ \\
\hline A levels/AS levels or equivalent & $6(23.1)$ \\
\hline O levels/GCSEs or equivalent & $8(30.8)$ \\
\hline CSEs or equivalent & $13(50.0)$ \\
\hline Employment & $1(3.8)$ \\
\hline Employed & $4(15.4)$ \\
\hline Unemployed & $1(3.8)$ \\
\hline Looking after home and/or family & $5(19.2)$ \\
\hline Student/trainee & $2(7.7)$ \\
\hline Retired because of PID & $2(7.7)$ \\
\hline Retired due to other reasons & $20(76.9)$ \\
\hline Marital status & $6(23.1)$ \\
\hline Married & $19(7.7)$ \\
\hline Unmarried/single & $17(65.4)$ \\
\hline IgG treatment ever and current & \\
\hline Intramuscular, intravenous, & \\
\hline and subcutaneous & \\
\hline Intravenous and subcutaneous & \\
\hline Intravenous & \\
\hline Subcutaneous & \\
\hline Hospital/at home & \\
\hline
\end{tabular}


Table 2. VAS and SF-36 scale values in all patients $(n=26)$ before and 6 months after PID-PEP. Values for median (range) are shown. Statistical significance was tested by Wilcoxon signed-rank test and $p$-values are given

\begin{tabular}{|c|c|c|c|}
\hline Variable & Before & After & $p$ value \\
\hline \multicolumn{4}{|l|}{ Summary measure } \\
\hline VAS & $68(10-98)$ & $76(35-98)$ & $0.002 *$ \\
\hline \multicolumn{4}{|l|}{ Summary measures SF-36 } \\
\hline $\begin{array}{l}\text { Physical component } \\
\text { summary (PCS) }\end{array}$ & $44(27-60)$ & $47(25-59)$ & 0.150 \\
\hline $\begin{array}{l}\text { Mental component } \\
\text { summary (MCS) }\end{array}$ & $36(20-53)$ & $43(13-55)$ & $0.042 *$ \\
\hline \multicolumn{4}{|l|}{ Dimensions SF-36 } \\
\hline Physical functioning (PF) & $85(40-100)$ & $85(40-100)$ & 0.302 \\
\hline Role-physical (RP) & $38(0-100)$ & $75(0-100)$ & 0.052 \\
\hline Bodily pain (BP) & $62(31-100)$ & $62(12-100)$ & 0.411 \\
\hline Social functioning (SF) & $63(25-100)$ & $75(13-100)$ & 0.228 \\
\hline Mental health (MH) & $64(36-76)$ & $68(28-80)$ & 0.081 \\
\hline Role-emotional (RE) & $83(0-100)$ & $100(0-100)$ & 0.214 \\
\hline Vitality (VT) & $48(20-65)$ & $55(15-70)$ & $0.025 *$ \\
\hline General health $(\mathrm{GH})$ & $41(15-82)$ & $45(5-87)$ & 0.252 \\
\hline
\end{tabular}

mittee of Hannover Medical School and all subjects gave written informed consent before participating.

\section{Evaluation of SF-36, visual analogue scale, and LQI before and at 6 months after PID-PEP}

SF-36 is a well-known, easy-to-use, validated, generic multi-item scale instrument for adults measuring eight health dimensions, i.e. 1) physical functioning (PF), 2) role limitations because of physical health problems (RP), 3) bodily pain (BP), 4) social functioning (SF), 5) general mental health $(\mathrm{MH}$, psychological distress and psychological well-being), 6) role limitations because of emotional problems (RE), 7) vitality (VT, energy/fatigue), and 8) general health perceptions (GH) [6]. The physical component summary (PCS) and mental component summary (MCS) were calculated based on the German population norm 1994 [12]. The SF-36 health dimensions and component summaries were transformed to a 0 (worst status) to 100 (best status) scale as described [13]. Furthermore, patients were asked to indicate their present health status on a visual analogue scale (VAS) ranging from 0 (worst status) to 100 (best status).

LQI has originally been developed for patients with PID receiving home-based intravenous immunoglobulin therapy [8]. It consists of 15 items assessing the percep- tion of the impact of IgG treatment on daily activities on a 7-point Likert scale ranging from extremely good (7) to extremely bad (1). The wording of the original LQI was changed slightly, i.e., the wording "IVIG treatment" was changed to "immunoglobulin treatment". The 15 items were summarized in four domains, i.e. I) treatment interference, II) therapy-related problems, III) therapy setting, and IV) treatment costs. Furthermore, the LQI index was calculated as a summary measure over all 15 items. The LQI domains and summary measure were transformed to a 0 (worst status) to 100 (best status) scale as described [7].

\section{Statistical analysis}

IBM SPSS software version 25 (IBM, Armonk, NY) was used in all statistical analyses. Differences before and at 6 months after PID-PEP were assessed by the Wilcoxon signed-rank test. A $p$-value of $<0.05$ was considered statistically significant in all analyses.

\section{Results}

\section{VAS and MCS values are significantly improved after PID-PEP}

Median (range) VAS values of present health status significantly increased from 68 (10-98) at baseline to 76 (35-98) 6 months after PID-PEP (Table 2, $p=0.002$ ). The SF-36 summary measure MCS significantly improved from 36 (20-53) to 43 (13-55) following the programme (Table 2, $p=0.042$ ). The SF-36 summary measure PCS numerically increased by 3 points; however, this improvement did not reach statistical significance (Table 2, $p=0.150$ ). Of the eight SF-36 dimensions, VT significantly improved from 48 (20-65) at baseline to 55 (15-70) at 6 months after PID-PEP (Table 2, $p=0.025$ ). Furthermore, there were nonsignificant $(p<0.10)$ improvements within role-physical (RP, baseline: 38 [0-100], at 6 months: 75 [0-100], $p=0.052$ ) and MH (baseline: 64 [36-76], at 6 months: 68 [28-80], $p=0.081$ ) (Table 2). No significant changes were observed within the SF-36 dimensions PF, BP, SF, role-emotional (RE), and GH (Table 2). PID patients at baseline showed numerically lower scores in all eight dimensions of the SF-36 as compared to a normal German population-representative control group (Fig. 1).

\section{LQI index, as well as LQI domains I and II, are significantly improved after PID-PEP}

Median (range) LQI index significantly increased from 77 (36-98) at baseline to 86 (44-99) 6 months after PID-PEP (Table 3, $p=0.008$ ). Furthermore, the LQI domains treatment interference (I) and therapy-related problems (II) significantly improved from 78 (39-100) to $88(39-100)(p=0.008)$ and $73(25-100)$ to $83(38-100)$ $(p=0.003)$, respectively (Table 3$)$. The LQI domains ther- 


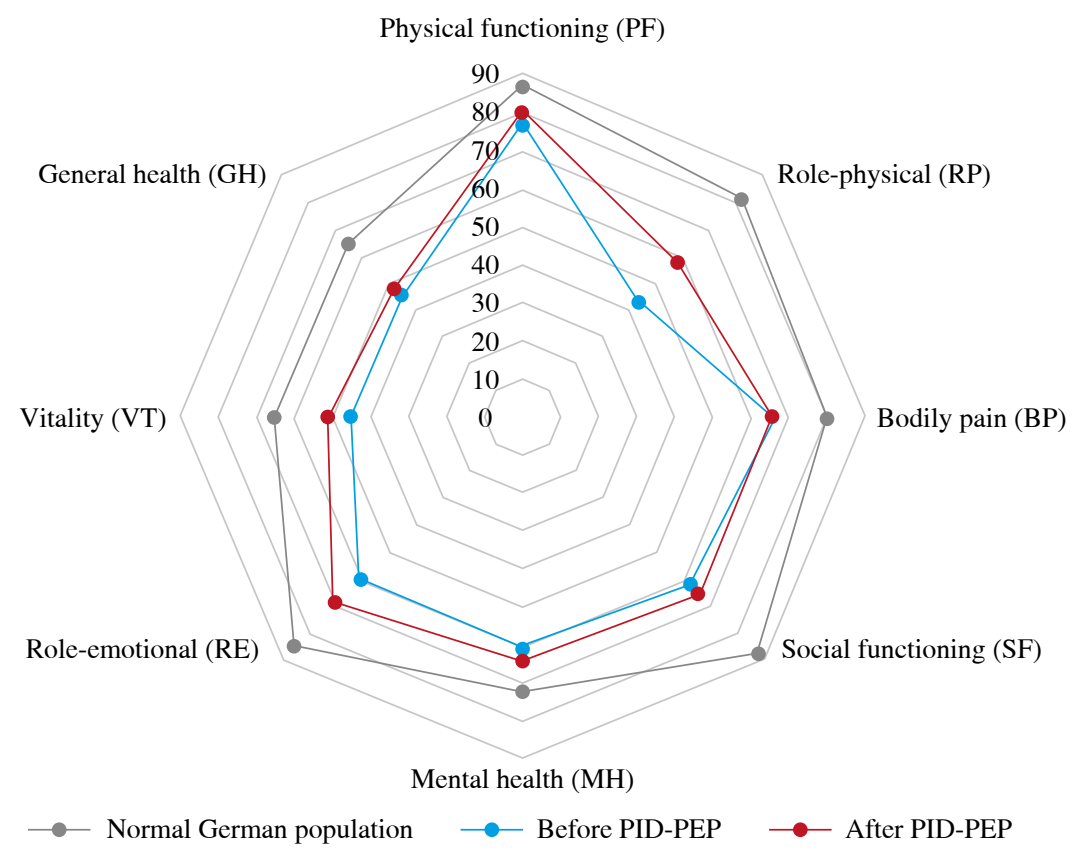

Fig. 1. Mean SF-36 dimensions in a German population-representative study [14], as well as in all patients $(n=26)$ before and 6 months after PID-PEP

apy setting (III) and treatment costs (IV) were unchanged after PID-PEP (Table 3).

\section{Discussion}

To the best of our knowledge, this is the first study to assess changes in QoL following participation of PID patients in a patient empowerment programme (PID-PEP). We report for the first time that the VAS score of present health status, MCS of the SF-36, and LQI index were significantly improved following PID-PEP.

All eight dimensions of the SF-36 were numerically lower in PID patients at baseline as compared to a normal German population-representative control group [14], indicating that QoL is significantly decreased in PID patients due to the severity of their chronic disease. In accordance with our findings, all eight SF-36 dimensions were also lower in Italian patients with common variable immunodeficiency disease (CVID) as compared to healthy subjects [4]. In the same CVID population, SF-36 scores for RP and $\mathrm{GH}$ were lower as compared to other chronic diseases including diabetes mellitus, cancer, chronic obstructive pulmonary disease, and mental disorders [4]. It is interesting to note in this context that mean RP values at baseline in our patient cohort were particularly low as compared to a normal German control group.

Of the eight SF-36 dimensions, six dimensions numerically improved and two were unchanged 6 months after PID-PEP as compared to baseline with VT, as well as MCS and VAS of present health status, reaching statistical significance. These findings suggest that improvements in QoL parameters are possible by a multidimensional PEP at least over a period of 6 months. It is important to consider in this context that QoL decreases over the natural course of a chronic disease. For CVID, Tabolli and co-workers demonstrated a significant decrease in five SF-36 dimensions, i.e. PF, BP, GH, SF, and RE, during an observation period of 6 years [4].

The LQI index significantly improved six months after PID-PEP as compared to baseline. The LQI is a wellknown tool to evaluate treatment satisfaction for IgG replacement therapy in four domains [7]. In our patients, the domains treatment interference with daily life activities

Table 3. LQI scale values in all patients $(n=26)$ before and 6 months after PID-PEP. Values for median (range) are shown. Statistical significance was tested by Wilcoxon signed-rank test and $p$-values are given

\begin{tabular}{lccc}
\hline Variable & Before & After & $\boldsymbol{p}$ value \\
\hline Summary measure & & & \\
\hline LQI index & $77(36-98)$ & $86(44-99)$ & $0.008^{*}$ \\
\hline Domains & & & \\
\hline Treatment interference (I) & $78(39-100)$ & $88(39-100)$ & $0.008^{*}$ \\
\hline $\begin{array}{l}\text { Therapy-related } \\
\text { problems (II) }\end{array}$ & $73(25-100)$ & $83(38-100)$ & $0.003^{*}$ \\
\hline Therapy setting (III) & $92(17-100)$ & $89(56-100)$ & 0.196 \\
\hline Treatment costs (IV) & $75(8-100)$ & $75(25-100)$ & 0.567 \\
\hline
\end{tabular}

$* p<0.05$ 
(I) and therapy-related problems (II) were significantly improved 6 months after PID-PEP. These improvements most likely reflect the focus of PID-PEP on motivation, education, and everyday transfer, next to practical issues of living with a chronic disease demanding lifelong therapy.

Well-being of patients has played a more central role in patient-centred clinical medicine in recent years [10]. This development does not substitute but adds to more traditional endpoints including mortality and morbidity. The present study provides evidence that PID-PEP improves the impact of chronic disease on health-related QoL in several domains over a period of 6 months. Since PID-PEP is not regularly covered by public and private health insurance companies, the present beneficial results should support informed decision making for health care providers. Cost-benefit analyses need to be performed to elucidate the cost-effectiveness of PID-PEP.

A strength of our study is the relatively long follow-up period of 6 months, implying that PID-PEP has sustainable effects on general and health-related QoL. Limitations include the lack of a control group, e.g. PID patients on a waiting list or only receiving written advice. Therefore, beneficial effects of PID-PEP on QoL cannot be proven by the present study; however, QoL parameters tend to decrease in CVID patients over time [4]. Due to the rarity of PID, the number of patients is limited. Since several general and health-related QoL parameters are significantly improved 6 months after PID-PEP, further studies in larger patient samples and with adequate control groups, as well as over longer-term follow-up, are warranted.

Taken together, it is shown for the first time that a patient empowerment programme for PID (PID-PEP) significantly improves general and health-related QoL. It needs to be evaluated in future studies whether the beneficial effects of PID-PEP are sustained over longer periods of time and whether repeated PID-PEP sessions further improve QoL outcome.

\section{Acknowledgments}

This study was supported by a grant to the PID-PEP working group by the Federal Ministry of Education and Research (BMBF), Germany, FKZ: 01GM0894, project A6. The funding body does not have any influence on the design of the study and collection, analysis, and interpretation of data, or in writing the manuscript. This work is dedicated to our patients and their families and we thank all study participants for their willingness to provide data for this research project.

The authors declare no conflict of interest.

\section{References}

1. Tangye SG, Al-Herz W, Bousfiha A, et al. (2020): Human inborn errors of immunity: 2019 update on the classification from the International Union of Immunological Societies Expert Committee. J Clin Immunol 40: 24-64.

2. McCusker C, Upton J, Warrington R (2018): Primary immunodeficiency. Allergy Asthma Clin Immunol 14 (Suppl 2): $142-152$.

3. Joshi AY, Iyer VN, Hagan JB, et al. (2009): Incidence and temporal trends of primary immunodeficiency: a population-based cohort study. Mayo Clin Proc 84: 16-22.

4. Tabolli S, Giannantoni P, Pulvirenti F, et al. (2014): Longitudinal study on health-related quality of life in a cohort of 96 patients with common variable immune deficiencies. Front Immunol 5: 605.

5. Brazier JE, Harper R, Jones NM, et al. (1992): Validating the SF-36 health survey questionnaire: new outcome measure for primary care. BMJ 305: 160-164.

6. Ware JE, Sherbourne CD (1992): The MOS 36-item shortform health survey (SF-36). I. Conceptual framework and item selection. Med Care 30: 473-483.

7. Nicolay U, Haag S, Eichmann F, et al. (2005): Measuring treatment satisfaction in patients with primary immunodeficiency diseases receiving lifelong immunoglobulin replacement therapy. Qual Life Res 14: 1683-1691.

8. Daly PB, Evans JH, Kobayashi RH, et al. (1991): Homebased immunoglobulin infusion therapy: quality of life and patient health perceptions. Ann Allergy 67: 504-510.

9. Jiang F, Torgerson TR, Ayars AG (2015): Health-related quality of life in patients with primary immunodeficiency disease. Allergy Asthma Clin Immunol 11: 27.

10. World Health Organization, Regional Office for Europe (2013): Health 2020: a European policy framework and strategy for the 21 st century. Available from: http://www.euro.who. int/_data/assets/pdf_file/0011/199532/Health2020-Long.pdf?ua $=1$ (cited 2020 Jan 10).

11. Pabst Science Publishers [ModuS Patient Education Programme for Primary Immunodeficiency, Version 2.0]. Available from: https://www.pabst-publishers.com/fileadmin/user_ upload/_modus_9783899678987/modus_9783958530751.pdf (cited 2020 Jan 10).

12. Bullinger M, Kirchberger I, Ware J (1995): The German SF-36 Health Survey. Z Gesundheitswissenschaft 3: 21-36.

13. Ware JE, Snow KK, Kosinski M, Gandek B (1993): SF-36 Health survey: manual and interpretation guide. Nimrod Press, Boston, MA.

14. Morfeld M, Bullinger M, Nantke J, Brähler E (2005): The version 2.0 of the SF-36 Health Survey: results of a population-representative study. Soz Praventivmed 50: 292-300. 


\section{Appendix}

\section{PID-PEP schedule}

(weekend course from Saturday 9 am to Sunday 3 pm)

\section{Module I) Team building and goal definition}

- Welcome, introduction of team members and participants.

- Expectations and goals.

- What is self-management and how can it improve daily life with a chronic condition?

\section{Module II) Education on chronic disease (PID)}

- How does the immune system work?

- What is immunodeficiency?

- PIDs with antibody deficiency.

- Genetics of PIDs.

Module III) Competences and motivation for longterm therapy

- Treatment options of PIDs.

- Treatment 1: Immunoglobulin replacement therapy (SCIG, IVIG); Production and safety of immunoglobulins; Exercises and practical aspects of home-based SCIG therapy.

- Treatment 2: Antibiotics; Supportive therapy (e.g. inhalation, physiotherapy); Vaccinations.

Module IV) Management of acute and emergency situations

- Managing severe infections.

- Managing severe immunoglobulin therapy-related effects.

\section{Module V) Empowerment and coping strategies}

- Balancing life with a long-term condition.

- Communication with family, friends, and colleagues.

- Coping with challenges and disease-related burden.

- Managing long-term medication.

- Recognising and managing setbacks.

- Celebrating stable conditions or improvements.

- Accepting chronic condition and long-term therapy.

Module VI) Everyday transfer of strategies learned

- Concluding session and open questions.

- Main goal to take home and transfer into everyday living.

- Feedback. 